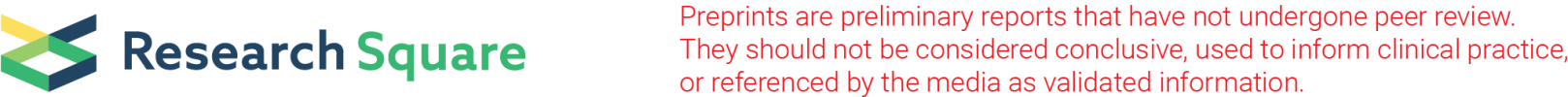

\section{Use Of Maximal Dosage Renin-Angiotensin- Aldosterone System Inhibitors In A Real Life Population Of Complicated Type 2 Diabetes - Contraindications And Opportunities}

C.M. Gant ( C.M.Gant@umcutrecht.nl )

Meander Medical Center

M.M. Oosterwijk

ZGT Hospital

S.H. Binnenmars

University of Groningen

G.J. Navis

University of Groningen

H. Haverkate

ZGT Hospital

S.J.L. Bakker

University of Groningen

G.D. Laverman

ZGT Hospital

Research Article

Keywords:

Posted Date: January 14th, 2022

DOI: https://doi.org/10.21203/rs.3.rs-1174424/v1

License: @ (i) This work is licensed under a Creative Commons Attribution 4.0 International License. Read Full License 


\section{Abstract}

\section{Objective}

Pharmacological inhibition of the renin-angiotensin-aldosterone-system (RAASi) is the cornerstone of hypertension treatment, renoprotection and secondary prevention of cardiovascular disease in patients with type 2 diabetes. Although there is a dose-dependent effect of RAASi with optimum protection when using maximal dose, little is known on actual use of maximal dosage RAASi in clinical practice. Here we investigate prevalence of maximal dosage RAASi, and contraindications for, optimizing RAASi dosage, in patients with complicated type 2 diabetes in a real-life clinical setting.

\section{Research Design and Methods}

We performed a retrospective analysis in 668 patients included in the DIAbetes and LifEstyle Cohort Twente (DIALECT). We grouped patients according to no RAASi, submaximal RAASi and maximal RAASi use. All potassium and creatinine measurements between January $1^{\text {st }} 2000$ and date of inclusion in DIALECT were extracted from patients files. We identified determinants of maximal RAASi use vs submaximal RAASi use with multivariate logistic regression analysis.

\section{Results}

Mean age was $64 \pm 10$ years and $61 \%$ were men. In total, 460 patients $(69 \%)$ used RAASi, and $30 \%$ used maximal RAASi. Maximal RAASi use was not statistically different between different indications for RAASi (i.e. hypertension, diabetic kidney disease, coronary heart disease and cerebrovascular disease; $P>0.05)$. Per patient, 2 [1-4] measurements of potassium and 20 [13-31] measurements of creatinine were retrieved, retrospective follow-up time was -3.0 [-1.4 to -5.7] years. Pre-baseline hyperkalemia $>5.0 \mathrm{mmol} / \mathrm{l}$ and acute kidney injury were found in 151 (23\%) patients and 119 patients (18\%), respectively. Determinants of maximal RAASi were prior acute kidney injury (OR 0.51 (0.30-0.87)), increased albuminuria (OR 1.89 (1.17-3.08)) and total number of used antihypertensives (OR 1.66 (1.33-2.06)).

\section{Conclusions}

Maximal dose RAASi is used in almost one third of complicated type 2 diabetes patients in a real-life setting. The prevalence of contraindications is considerable, but relative in nature, suggesting that it is worthwhile to explore strategies aimed at maximizing RAASi while circumventing the alleged contraindications.

\section{Introduction}

Renin-angiotensin aldosterone system inhibition (RAASi) is indicated for several conditions related to type 2 diabetes. Guidelines recommend RAASi as the first step treatment for hypertension in type 2 diabetes, for renoprotection in patients with diabetic kidney disease (DKD), and for secondary prevention in patients with established cardiovascular disease (CVD) (1-3). However, real-life data on the actual use, and dosing, of RAASi in high-risk patients are scarce. 
Studies have consistently demonstrated RAASi treatment improves all stages of albuminuria and reduces endstage kidney disease, cardiovascular events and death (4-8). The beneficial effects of RAASi are in part attributable to the blood-pressure lowering properties of RAASi, but RAASi also limits target organ damage development through blood pressure independent pathways, thereby further reducing risk of adverse renal events, cardiovascular events and all-cause mortality (9). However, the ongoing high incidence of major adverse renal and cardiovascular events demonstrates the urgency to improve secondary prevention in at-risk patients.

To achieve optimum treatment effects of RAASi on blood pressure, albuminuria and target organ damage, it is vital that patients receive maximum effective dosage RAASi (10-14). The dose-response curves of RAASi differ per desired effect. For instance, increasing RAASi from low to maximum dosage may not result in further blood pressure lowering(10). However, for reducing albuminuria and for target organ protection, maximizing RAASi dose is of the utmost importance, as it reduces hard endpoints such as cardiovascular and all-cause mortality $(10,14)$. In clinical practice, increasing RAASi dose may be hampered by adverse events such as hyperkalemia, acute kidney injury (AKI) and hypotension, therefore limiting renal and cardiovascular prevention (15).

So far, real-word data on the penetrance of the maximum dose RAASi prescription and contraindications for increasing RAASi dosage are scarce. Therefore, here we investigate use of, and documented contraindications for, maximum dosage RAASi in a real-life setting of type 2 diabetes patients.

\section{Methods}

We performed a retrospective analysis using data from the DIAbetes and LifEstyle Cohort Twente (DIALECT). The study population and study procedures of DIALECT have been described previously (16). DIALECT is an observational cohort study in patients with type 2 diabetes, which was designed to study lifestyle and pharmacological factors and their associations with clinical outcomes. The study has been approved by local institutional review boards (METC-Twente, NL57219.044.16; METC-Groningen, 1009.68020), is registered in the Netherlands Trial Register (NTR trial code 5855) and is performed according to the guidelines of good clinical practice and the declaration of Helsinki as revised in 2008. All participants signed an informed consent form before participation.

\section{Setting}

Between September 2009 and May 2019, a total of 668 patients with type 2 diabetes were included in DIALECT (16). DIALECT was performed in the outpatient clinic internal medicine of the Ziekenhuis Groep Twente (ZGT) Hospital, Almelo and Hengelo, the Netherlands. The ZGT Hospital is a secondary care center for diabetes treatment. In the Netherlands, referral criteria to secondary health care are as follows: inability to achieve adequate glycemic control with oral antidiabetic drugs or a standard insulin regimen, overt nephropathy (macroalbuminuria and/or estimated glomerular filtration rate (eGFR) below $60 \mathrm{ml} / \mathrm{min} / 1.73 \mathrm{~m}^{2}$ ), or multiple cardiovascular complications.

\section{Participants}


All patients, aged $18+$ years, visiting the internal medicine outpatient clinic for type 2 diabetes treatment were eligible for the study (16). Exclusion criteria were inability to understand the informed consent procedure, insufficient command of the Dutch language, or renal replacement therapy. Eligible patients were selected from the electronic patient file and contacted by phone.

\section{Variables}

At the clinic, sociodemographic characteristics, medical history, lifestyle behaviors, and current medications were registered and anthropometric dimensions were measured using standard procedures (16). Medical history was additionally reviewed in the hospital electronic patient files independently by three different physician researchers. Medication use was extensively verified by data on drug prescriptions and drug deliveries provided by local pharmacies. In case of mismatching, the pharmacy delivery information was considered appropriate above the electronic patient file data, as pharmacy dispensing data more closely reflect the drugs the patient actually receives.

Coronary heart disease (CHD) was defined as the presence of one of the following items in medical history: physician diagnosed unstable angina pectoris, myocardial infarction, percutaneous coronary intervention, or coronary artery bypass graft. Cerebrovascular disease (TIA/CVA) was defined as a history of transient ischemic attack or cerebrovascular accident.

Blood pressure was measured in a supine position by an automated device (Dinamap®; GE Medical Systems, Milwaukee, $\mathrm{WI}$ ) for $15 \mathrm{~min}$ with a $1 \mathrm{~min}$ interval. The mean systolic and diastolic pressure of the final three measurements was used for further analysis.

Blood was drawn from venipuncture, and morning void urine and 24-hour urine was collected as prescribed previously (16). Data on dietary sodium intake was derived from the 24 -hour urinary sodium excretion.

To study contraindications for maximal RAASi dosing we collected extensive retrospective data on serum potassium levels and creatinine levels from the hospital's laboratory system. Potassium and creatinine data were extracted for all patients files using a query for all serum potassium and creatinine measurements registered between 01-01-2000 and date of inclusion in DIALECT.

\section{Targets and definitions}

Patients were categorized in three groups according to RAAS inhibition use: patients who did not use RAASi (noRAASi), patients who used RAASi but not in the maximum dose (smRAASi) and patients who used maximal dose RAASi (mRAASi). Maximal daily dose of RAASi was based on existing literature, and is described in appendix 1 .

As the use of direct renin inhibitors (i.e., aliskiren) and mineralocorticoid receptor antagonists (i.e., spironolactone and eplerenone) has not been well defined for renoprotection and cardiovascular prevention in patients with type 2 diabetes, we did not include these agents in the definition of maximal dosage RAASi.

Indications for RAASi were categorized as following: secondary prevention in patients with chronic kidney disease (i.e. albuminuria and/or eGFR $<60 \mathrm{ml} / \mathrm{min} / 1.73 \mathrm{~m}^{2}$ ), hypertension, stroke, heart failure, and $\mathrm{CHD}(1,2)$. 
Hyperkalemia was defined as serum potassium $>5.0 \mathrm{mmol} / \mathrm{l}$, and serious hyperkalemia as potassium $\geq 6.0$ $\mathrm{mmol} / \mathrm{l}$.

AKI was defined according to KDIGO guidelines: stage 1, rise in serum creatinine 1.5-1.9 times of previous value; stage 2, rise in serum creatinine of 2-2.9 times of previous value; stage 3 , rise in serum creatinine 3 times or higher of previous value.

Moderately increased albuminuria was defined as $>30 \mathrm{mg} / 24 \mathrm{~h}$ albumin excretion, or increased albumin/creatinine ratio ( $>2.5 \mathrm{mg} / \mathrm{mmol}$ for men and $>3.5 \mathrm{mg} / \mathrm{mmol}$ for women) in morning void urine when $24 \mathrm{~h}$ hour urine was missing. According to the KDIGO guidelines, target blood pressure was $\leq 140 / 90 \mathrm{mmHg}$ for patients without albuminuria, and $\leq 130 / 80 \mathrm{mmHg}$ for patients with albuminuria.

\section{Statistics}

All statistical analyses were performed using Statistical Package for the Social Sciences (IBM, Chicago, IL, USA), version 22.0. Normality of data was assessed by visually inspecting the frequency histograms. Normally distributed data were presented as mean \pm SD. Skewed variables were expressed as median [interquartile range]. Dichotomous variables were presented in number and percentage. Cases with missing data were excluded from the respective analyses.

Differences between the three RAASi groups (noRAASi, smRAASi, mRAASi) were tested using one-way analysis of variance with bonferroni post hoc analyses (normally distributed), Kruskal-Wallis (skewed), or X2-test (categorical).

We investigated determinants of maximal RAASi vs submaximal RAASi using multivariate logistic regression analyses. Candidates for the model were assessed using univariate logistic regression and literature research. The multivariate model was constructed using backward step analysis. For variables with high collinearity, one variable was chosen for inclusion in the multivariate model. Additionaly, we studied determinants of RAASi use versus no RAASi use using the same analysis.

\section{Results}

Mean age of the 668 patients included in the study was $64 \pm 10$ years, $61 \%$ of patients were men, and median duration of type 2 diabetes was 12 [7-19] years (Table 1). Prevalence of co-morbidity was high as 35\% $(n=231)$ of patients had albuminuria and $36 \%(n=242)$ of patients had one or more macrovascular complications. 
Table 1

- Baseline characteristics of patients included in DIALECT, divided by dosage groups of renin-angiotensinaldosterone system inhibition

\begin{tabular}{|c|c|c|c|c|c|c|}
\hline & & $\begin{array}{l}\text { Total } \\
\text { population }\end{array}$ & No RAASi & $\begin{array}{l}\text { Submaximal } \\
\text { RAASi }\end{array}$ & $\begin{array}{l}\text { Maximum } \\
\text { RAASi }\end{array}$ & P-value \\
\hline & $\mathrm{n}$ & $n=668$ & $\begin{array}{l}\mathrm{n}=208 \\
(31)\end{array}$ & $\mathrm{n}=262(39)$ & $\begin{array}{l}n=198 \\
(30)\end{array}$ & \\
\hline Age years & 668 & $64 \pm 10$ & $61 \pm 12$ & $65 \pm 9 *$ & $65 \pm 9 *$ & $<0.001$ \\
\hline Gender men, n (\%) & 668 & $405(61)$ & $131(63)$ & $147(56)^{\star}$ & $127(64) \star$ & 0.15 \\
\hline $\begin{array}{l}\text { Body mass index, } \\
\mathrm{kg} / \mathrm{m} 2\end{array}$ & 666 & $32.6 \pm 5.9$ & $31.2 \pm 5.9$ & $33.1 \pm 5.6$ & $33.5 \pm 6.1$ & $<0.001$ \\
\hline SBP/DBP, mmHg & $666 / 666$ & $\begin{array}{l}134 / 74 \pm \\
16 / 10\end{array}$ & $\begin{array}{l}140 / 78 \pm \\
16 / 9\end{array}$ & $\begin{array}{l}138 / 74 \pm \\
16 / 10 *\end{array}$ & $\begin{array}{l}141 / 76 \pm \\
16 / 9\end{array}$ & $0.16 / 0.02$ \\
\hline $\begin{array}{l}\mathrm{SBP}<100 \mathrm{mmHg}, \mathrm{n} \\
(\%)\end{array}$ & 666 & $9(1)$ & $2(1)$ & $4(2)$ & $3(2)$ & 0.85 \\
\hline $\begin{array}{l}\text { Heart frequency, } \\
\text { beats/min }\end{array}$ & 657 & $73 \pm 13$ & $73 \pm 12$ & $73 \pm 13$ & $72 \pm 12$ & 0.46 \\
\hline Smoker, yes/former (\%) & 668 & $\begin{array}{l}106 / 345 \\
(16 / 52)\end{array}$ & $\begin{array}{l}43 / 106 \\
(21 / 51)\end{array}$ & $\begin{array}{l}38 / 126 \\
(15 / 49)\end{array}$ & $\begin{array}{l}25 / 113 \\
(13 / 58)\end{array}$ & 0.05 \\
\hline $\begin{array}{l}\text { Years since type } 2 \\
\text { diabetes diagnosis, } \\
\text { years }\end{array}$ & 668 & $12[7-19]$ & $11[5-17]$ & $12[7-19]$ & $14[8-20]$ & 0.06 \\
\hline $\begin{array}{l}\text { Serum } \mathrm{HbA} 1 \mathrm{c} \\
\mathrm{mmol} / \mathrm{mol}\end{array}$ & 666 & $58 \pm 12$ & $57 \pm 12$ & $59 \pm 13$ & $59 \pm 12$ & 0.22 \\
\hline Insulin use, n (\%) & 668 & $427(64)$ & $125(60)$ & $175(67)$ & $127(64)$ & 0.32 \\
\hline $\begin{array}{l}\text { Diabetic kidney disease, } \\
\text { n (\%) }\end{array}$ & 664 & $314(47)$ & $70(34)$ & $127(49)$ & $117(59)$ & $<0.001$ \\
\hline $\begin{array}{l}\text { eGFR }<60 \\
\mathrm{ml} / \mathrm{min} / 1.73 \mathrm{~m} 2, \mathrm{n}(\%)\end{array}$ & 668 & $176(26)$ & 40 (19) & $72(28)$ & $64(32)$ & 0.01 \\
\hline $\begin{array}{l}\text { eGFR <30 } \\
\mathrm{ml} / \mathrm{min} / 1.73 \mathrm{~m} 2, \mathrm{n}(\%)\end{array}$ & 667 & $25(4)$ & $7(3)$ & $11(4)$ & $7(4)$ & 0.88 \\
\hline $\begin{array}{l}\text { Moderately increased } \\
\text { albuminuria, } \mathrm{n}(\%)\end{array}$ & 660 & $231(35)$ & $52(25)$ & $89(34)$ & $90(46)$ & $<0.001$ \\
\hline Retinopathy, n (\%) & 658 & $150(23)$ & $32(16)$ & $64(25)$ & $54(27)$ & 0.02 \\
\hline Polyneuropathy, n (\%) & 666 & $260(39)$ & $61(30)$ & $116(44)$ & $83(42)$ & 0.003 \\
\hline $\begin{array}{l}\text { Coronary heart disease, } \\
\text { n (\%) }\end{array}$ & 667 & $153(23)$ & 40 (19) & $65(25)$ & $48(24)$ & $<0.001$ \\
\hline
\end{tabular}

SBP, systolic blood pressure. DBP, diastolic blood pressure. eGFR, estimated glomerular filtration rate. AKI, acute kidney injury 


\begin{tabular}{|c|c|c|c|c|c|c|}
\hline & & $\begin{array}{l}\text { Total } \\
\text { population }\end{array}$ & No RAASi & $\begin{array}{l}\text { Submaximal } \\
\text { RAASi }\end{array}$ & $\begin{array}{l}\text { Maximum } \\
\text { RAASi }\end{array}$ & P-value \\
\hline $\begin{array}{l}\text { Cerebrovascular } \\
\text { disease, } \mathrm{n}(\%)\end{array}$ & 667 & $80(12)$ & $16(8)$ & $41(16)$ & $23(12)$ & 0.06 \\
\hline \multicolumn{7}{|l|}{ Heart failure } \\
\hline $\begin{array}{l}\text { Reduced ejection } \\
\text { fraction, } \mathrm{n}(\%)\end{array}$ & 433 & $13(3)$ & $0(0)$ & $7(4)$ & $6(5)$ & 0.16 \\
\hline $\begin{array}{l}\text { Preserved ejection } \\
\text { fraction, } \mathrm{n}(\%)\end{array}$ & 433 & $11(3)$ & $2(2)$ & $6(3)$ & $3(2)$ & \\
\hline \multicolumn{7}{|c|}{ Pharmacological management } \\
\hline $\begin{array}{l}\text { Angiotensin converting } \\
\text { enzyme inhibitors, } n(\%)\end{array}$ & 667 & $201(30)$ & $0(0)$ & $138(53)$ & $63(32)$ & \\
\hline $\begin{array}{l}\text { Angiotensin II receptor } \\
\text { blockers, n (\%) }\end{array}$ & 668 & $264(40)$ & $0(0)$ & $127(49)$ & $138(70)$ & \\
\hline $\begin{array}{l}\text { Aldosterone antagonist, } \\
\mathrm{n}(\%)\end{array}$ & 668 & $64(10)$ & $16(8)$ & $22(8)$ & $26(13)$ & 0.13 \\
\hline Renin inhibitor, n (\%) & 668 & $6(1)$ & $5(2)$ & $1(0)$ & $0(0)$ & 0.02 \\
\hline Thiazide diuretics, n (\%) & 668 & $201(30)$ & $24(12)$ & $85(32)$ & $92(47)$ & $<0.001$ \\
\hline Loop diuretics, n (\%) & 668 & $121(18)$ & $19(9)$ & $65(25)$ & $37(19)$ & $<0.001$ \\
\hline Beta blockers, n (\%) & 668 & $317(47)$ & $69(33)$ & $128(49)$ & $120(61)$ & $<0.001$ \\
\hline $\begin{array}{l}\text { Calcium antagonists, } \mathrm{n} \\
(\%)\end{array}$ & 668 & $171(26)$ & $31(15)$ & $56(21)$ & $84(42)$ & $<0.001$ \\
\hline $\begin{array}{l}\text { Total number of } \\
\text { antihypertensives }\end{array}$ & 667 & $2[1-3]$ & $0[0-2]$ & $2[2-3]$ & $3[2-4]$ & $<0.001$ \\
\hline $\begin{array}{l}\text { Blood pressure on } \\
\text { target, } \mathrm{n}(\%)\end{array}$ & 658 & $273(42)$ & $86(42)$ & $98(38)$ & $89(46)$ & 0.18 \\
\hline $\begin{array}{l}\text { Hypertension requiring } \\
4+\text { drugs, } n(\%)\end{array}$ & 657 & $173(26)$ & $7(3)$ & $74(28)$ & $92(48)$ & $<0.001$ \\
\hline \multicolumn{7}{|l|}{ Serum biochemistry } \\
\hline Serum sodium, mmol// & 666 & $139 \pm 3$ & $139 \pm 3$ & $139 \pm 3$ & $139 \pm 3$ & 0.16 \\
\hline $\begin{array}{l}\text { Serum potassium, } \\
\mathrm{mmol} / \mathrm{l}\end{array}$ & 667 & $4.1 \pm 0.4$ & $4.1 \pm 0.4$ & $4.1 \pm 0.4$ & $4.1 \pm 0.5$ & 0.74 \\
\hline $\begin{array}{l}\text { Hyperkalemia, } \mathrm{n} \\
(\%)\end{array}$ & 667 & $12(2)$ & $3(1)$ & $5(2)$ & $4(4)$ & 0.90 \\
\hline
\end{tabular}

SBP, systolic blood pressure. DBP, diastolic blood pressure. eGFR, estimated glomerular filtration rate. AKI, acute kidney injury 


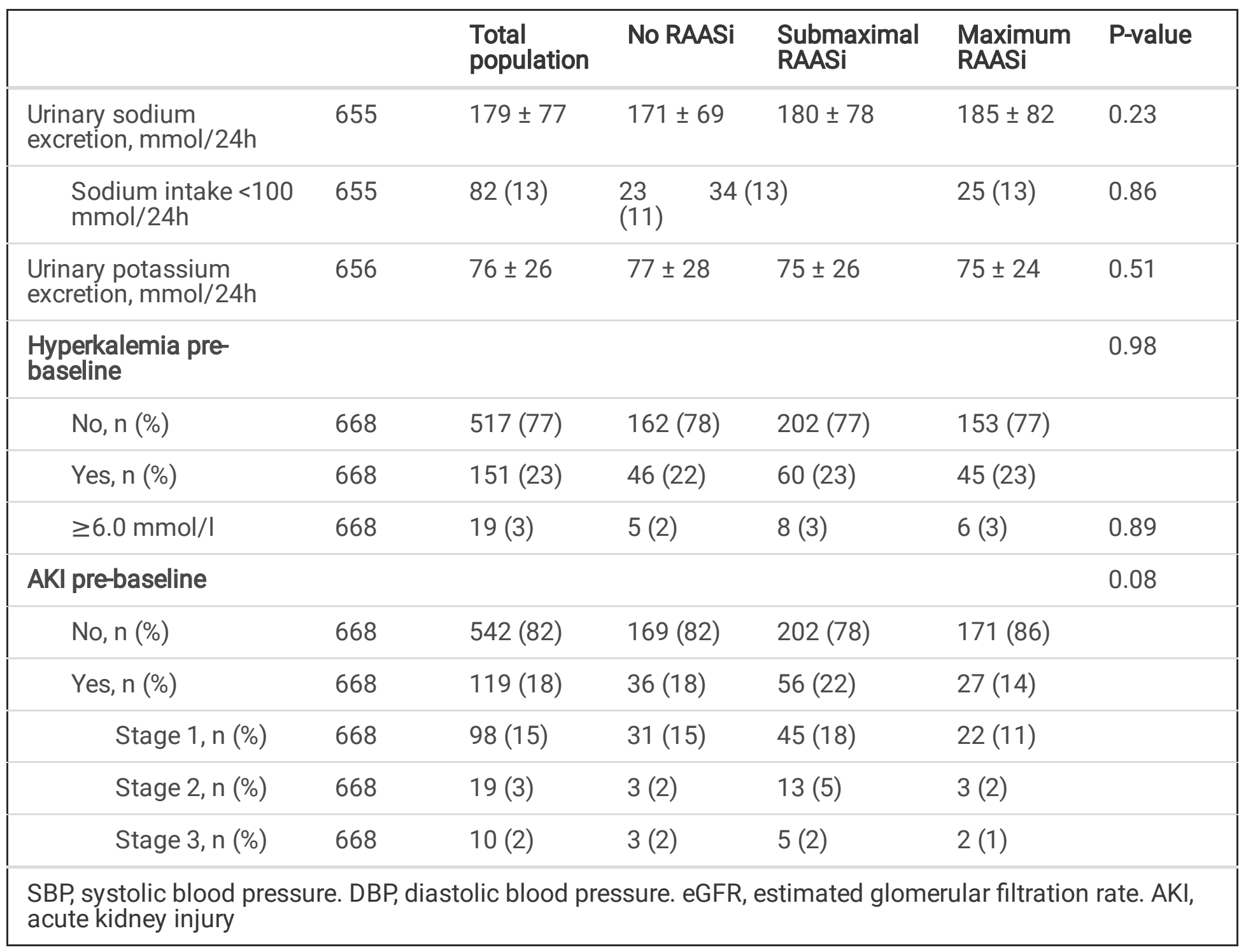

In total, $460(69 \%)$ of the patients used RAASi, and 198 (30\%) of patients used RAASi in the maximal dosage. In general, patients on either smRAASi or mRAASi were older ( $65 \pm 9$ and $65 \pm 9$ years) than noRAASi (61 \pm 12 years; $P<0.001)$ and had a higher body mass index $\left(33.1 \pm 5.6\right.$ and $\left.33.5 \pm 6.1 \mathrm{~kg} / \mathrm{m}^{2}\right)$ than noRAASi $(31.2 \pm 5.9$ $\left.\mathrm{kg} / \mathrm{m}^{2} ; \mathrm{P}<0.001\right)$. The prevalence of diabetic kidney disease was highest in patients with maximum dosage RAASi ( $59 \%$ vs $49 \%$ in smRAASi and $34 \%$ in noRAASi; $P<0.001)$. Prevalence of CHD ( $25 \%$ vs $24 \%$ ) and TIA/CVA (12 vs 16\%) did not materially differ between smRAASi and mRAASi. Interestingly, the median total number of used antihypertensive agents was highest in the mRAASi group (3 [2-4] vs smRAAsi 2 [2-3] and noRAASi 0 [02]; $P<0.001)$. There was no difference in baseline serum potassium between the groups $(P=0.74)$. Urinary sodium excretion was not statistically significantly different between the groups $(P=0.23)$. In total, $13 \%$ of patients $(n=82)$ adhered to the guideline of $<100 \mathrm{mmol}$ sodium intake per day (corresponding with $<6$ gram sodium chloride), and this proportion did not differ between the groups $(p=0.86)$.

When investigating RAASi dosage per indication for RAASi (i.e., hypertension, DKD, CHD and TIA/CVA), we found that the proportion of patients on mRAASi was highest in those with the combination of hypertension, DKD and CHD (42\%), although differences were not statistically significant ( $P>0.05$; see figure 1$)$. There were no differences in smRAASi $(4 \%)$ or mRAASi $(5 \%)$ use in patients with heart failure with reduced ejection fraction (Table 1). 
To investigate potential contraindications for mRAASi, we studied hyperkalemia and AKI that occurred before the baseline visit (Table 1). In total, there were 12,688 plasma potassium and 16,544 plasma creatinine measurements performed between 2000 and baseline assessments of the DIALECT cohort. Per individual patient, these were a median 2 [1-4] measurements of potassium and a median 20 [13-31] measurements of creatinine. Median retrospective follow-up time was -3.0 [-1.4 to -5.7] years.

We found that 151 (23\%) of patients had experienced hyperkalemia $>5.0 \mathrm{mmol} / \mathrm{l}$ at least once, 19 (3\%) of whom at least once had a plasma potassium $\geq 6.0 \mathrm{mmol} / \mathrm{l}$. Of the 33 patients with more than 5 previous hyperkalemic events, 8 were noRAASi (24\%), 14 were smRAASi (42\%) and 11 were mRAASi (33\%; figure 2A). In patients with 1-2 or with 3 prebaseline hyperkalemic events, the distribution of noRAASi, smRAASi and mRAASi was not statistically different $(P=0.90)$. There were no differences in the prevalence of serious hyperkalemia $\geq 6.0 \mathrm{mmol} / \mathrm{l}$ between the RAASi categories (Table 1). At the time of the baseline visit, the actual prevalence of hyperkalemia was low: only $2 \%$ of the patients $(n=12)$ had a plasma potassium concentration $>5.0 \mathrm{mmol} / \mathrm{l}$, the prevalence was not different in the RAASi groups.

An episode of AKI in the pre-baseline period was noted in 119 patients (18\%), of which 10 patients (2\%) had a stage $3 \mathrm{AKI}$. Further inspection of the electronic patient files demonstrated that none of the AKI events were related to initiation or dosage increase of RAASi. Of the 5 patients that had experience 3 pre-baseline AKIs, none used mRAASi, 4 used smRAASi, and 1 used noRAASi (figure 2B). There was a statistically non-significant trend towards lower use of mRAASi in those with 2 pre-baseline AKIs (20\%), compared to mRAASi use in those with $1(24 \%)$ or no (32\%) prior AKIs $(P=0.17)$. At baseline, the prevalence of severe renal impairment (eGFR <30 $\left.\mathrm{ml} / \mathrm{min} / 1.73 \mathrm{~m}^{2}\right)$ as a potential contraindication was low $(\mathrm{n}=25 ; 4 \%)$. Few patients had hypotension as a contraindication for increasing RAASi dosage (systolic blood pressure $<100 \mathrm{mmHg} ; \mathrm{n}=9 ; 1 \%$ ).

To identify independent determinants of mRAASi compared to smRAASi, we performed univariate logistic regression analyses followed by multivariate logistic regression analysis. In univariate analysis (Table 2), the presence of moderately increased albuminuria is significantly associated with mRAASi (OR 1.67 (1.14-2.45)). Also, the total number of used antihypertensives was associated with mRAASi (OR 1.49 (1.26-1.77)). The number of prior AKIs was a negative predictor of mRAASi (OR 0.60 (0.40-0.89)). In multivariate analysis (Table 2), moderately increased albuminuria (OR 1.89 (1.17-3.08)), the total number of used antihypertensives (OR 1.66 (1.33-2.06)) and number of prior AKIs (OR 0.51 (0.30-0.87)) remained strong statistically independent predictors of mRAASi. Furthermore, TIA/CVA (OR 0.43 (0.21-0.90)) appeared as a negative predictor of mRAASi use. eGFR<60 ml/min/ $1.73 \mathrm{~m}^{2}$ or frequency of pre-baseline hyperkalemia were not associated with mRAASi compared to smRAASi. 
Table 2

- Logistic regression analysis on determinants of maximal RAASi vs submaximal RAASi

\begin{tabular}{|c|c|c|}
\hline & Univariate & Multivariate \\
\hline & $\operatorname{Exp}(B)(95 \% \mathrm{Cl})$ & \\
\hline Age years & $1.00(0.98-1.03)$ & $\ldots$ \\
\hline Gender, women vs men & $0.72(0.49-1.04)$ & $\ldots$ \\
\hline Body mass index, $\mathrm{kg} / \mathrm{m}^{2}$ & $1.01(0.98-1.05)$ & $\ldots$ \\
\hline SBP, mmHg & $1.01(0.99-1.02)$ & $\ldots$ \\
\hline DBP, mmHg & $1.00(0.99-1.02)$ & $\ldots$ \\
\hline Heart frequency, beats/min & $0.99(0.98-1.01)$ & $\ldots$ \\
\hline Smokers vs non-smokers & $0.93(0.55-1.58)$ & $\ldots$ \\
\hline Years since type 2 diabetes diagnosis, years & $1.02(1.00-1.04)$ & $\ldots$ \\
\hline Serum $\mathrm{HbA} 1 \mathrm{c}, \mathrm{mmol} / \mathrm{mol}$ & $0.99(0.98-1.01)$ & \\
\hline Insulin use & $0.89(0.60-1.31)$ & \\
\hline eGFR $<60 \mathrm{ml} / \mathrm{min} / 1.73 \mathrm{~m}^{2}$ & $0.79(0.53-1.19)$ & \\
\hline Albuminuria & $1.67(1.14-2.45)$ & $1.89(1.17-3.08)$ \\
\hline Retinopathy & $1.14(0.75-1.74)$ & $\ldots$ \\
\hline Polyneuropathy & $0.90(0.62-1.31)$ & $\ldots$ \\
\hline Coronary heart disease & $0.97(0.63-1.49)$ & $\ldots$ \\
\hline Cerebrovascular disease & $0.71(0.41-1.22)$ & $0.43(0.21-0.90)$ \\
\hline Total number of antihypertensives & $1.49(1.26-1.77)$ & $1.66(1.33-2.06)$ \\
\hline Prebaseline hyperkalemia $>6 \mathrm{mmol} / \mathrm{L}$ & $0.99(0.34-2.91)$ & $\ldots$ \\
\hline \multicolumn{3}{|l|}{ Number of prebaseline hyperkalemia events } \\
\hline 0 & ref & $\ldots$ \\
\hline $1-2$ times & $0.96(0.43-2.18)$ & \\
\hline 3-5 times & $0.91(0.35-2.33)$ & $\ldots$ \\
\hline$>5$ times & $1.04(0.32-3.40)$ & $\ldots$ \\
\hline Number of prebaseline acute kidney injury events & $0.60(0.40-0.89)$ & $0.51(0.30-0.87)$ \\
\hline
\end{tabular}


In an additional analysis we investigated determinants of RAASi use versus no RAASi use, here we found that albuminuria or polyneuropathy were the strongest predictors of RAASi use (supplementary Table 1). Prior AKI or hyperkalemia were not associated with the use of RAASi.

\section{Discussion}

We investigated maximum dosage RAASi use in a real-life routine care setting of complicated type 2 diabetes patients. Only a third of these patients receive maximum dose treatment, which is in line with a previously reported proportion of 19-26 percent, found in a population of 195,327 patients with chronic kidney disease, diabetes and/or heart failure (15).

When we tried to find the reasons for not using maximum RAASi dosage, it appeared that a considerable number of patients (23\%) had a previous history of at least one measurement of hyperkalemia but this percentage was not different between the maximal (mRAASi) and submaximal (smRAASi) group. This, and the finding that the actual prevalence of hyperkalemia at baseline was $2 \%$, suggests that other considerations must play a role, e.g., a clinical judgement of likelihood of reoccurrence. It should be emphasized however, that reducing the RAASi dose is not necessarily the primary option to prevent hyperkalemia. Reducing dietary potassium intake and/or the use of oral potassium binders are effective measures that should be taken into consideration as a first step in this respect.

Another potential factor limiting the use of RAASi is the occurrence of acute kidney injury (AKI) prior to baseline. We found that almost $20 \%$ of the patients had a history of AKI and that the occurrence of which was strongly associated with the use of submaximal dose RAASi instead of maximal dose. The occurrence of AKI, regardless of the cause, is often an important reason to pause RAASi to restore glomerular filtration pressure. However, in follow-up after AKI, persistent RAASi use is associated with decreased mortality, although the risk for hospitalization due to renal causes is higher(17). As in our population, AKI prior to baseline did not occur after initiation or increasing dose of RAASi, and as current severe renal impairment eGFR $<30 \mathrm{ml} / \mathrm{min} / 1.73 \mathrm{~m}^{2}$ was rare (4\% of patients), carefully monitored increase of RAASi dosage in patients with previous AKI could be attempted.

Finally, low blood pressure can be a limiting factor for maximal dosing of RAASi. We found that $1 \%$ of the patients had documented hypotension defined as a systolic blood pressure below $100 \mathrm{mmHg}$. Notably, the prevalence of difficult to treat hypertension was very high, as the majority of patients did not reach the target blood pressure (58\%), and the median number of used antihypertensive agents was 2 . When patients use an antihypertensive drug with limited renal and cardiovascular preventive effects, compared to RAASi, it could be considered to cease these agents, in order to initiate, or increase, RAASi therapy.

Taken together, there is ample opportunity to increase the dosage of RAASi in this population of patients with advanced type 2 diabetes, as 1) hyperkalemia occurs in a minority of patients and is manageable with alternative strategies; 2) hypotension is rare; 3) Previous AKI is relatively highly frequent and important to consider when maximizing RAASi treatment, but should not be a reason to forego RAASi optimization.

Because this was a retrospective and observational study, we cannot guarantee $100 \%$ correct adverse effects documentation in the patient files. On the other hand, to our knowledge, it is extremely rare that patients have a 
contraindication limiting the use of any RAASi, and if this were the case, it would be extremely unlikely that this would not be documented in the files.

Apart from contraindications, which are patient related factors, physicians may have preferences -in general and in specific situations- that are likely to influence the penetrance of maximal RAASi dosing. First, alternative antihypertensive drug classes could be preferred in certain situations due to competing indications, e.g., beta blockers or calcium channel blockers when CHD coincides with type 2 diabetes. Our finding that TIA/CVA was a negative predictor of mRAASi compared to smRAASi, could be a reflection of such a phenomenon. The guidelines on secondary stroke prevention recommend lowering blood pressure with combination therapy of multiple antihypertensives in a low dose, rather that maximizing RAASi(18). Also, in hypertension treatment, an approach of combining drugs at submaximal doses has been advocated as a strategy to treat hypertension with less side-effects(19). This strategy does not seem to be adopted here, as is indicated by our finding that the number of used antihypertensives was strongly positively associated with mRAASi.

The most important patient characteristics associated with mRAASi were the presence of moderately increased albuminuria, and difficult to treat hypertension. The former was an expected finding, as albuminuria is a very important indication for initiation of RAASi. However, even in patients with albuminuria, mRAASi use was still $46 \%$, although ideally all patients should receive mRAASi, as it is associated with a decrease in hard cardiovascular and renal endpoints(11). Our finding that difficult to treat hypertension is strongly associated with mRAASi suggests that clinicians often do increase RAASi dosage in the scope of blood pressure treatment. Surprisingly, coronary heart disease, which is a very important indication for RAASi, was not associated with mRAASi compared to smRAASi. Although these patients also use other agents which act on blood pressure and possibly renal function, our data demonstrated that the prevalence of unequivocal contraindications for increasing RAASi dosage is low, which suggests that there is enough possibility to increase RAASi dosage.

Reduction of dietary sodium intake is an additional well-established method to increase RAASi effectivity(20). In our population, only $13 \%$ of patients adhered to the guideline on sodium intake of $<100 \mathrm{mmol} /$ day. Therefore, next to increasing RAASi dosage, reducing dietary sodium could be a good strategy to increase RAASi efficacy.

This study has several strengths. First, it represents a real-life population of patients with complicated type 2 diabetes, and therefore provides important insights on implementation of pharmacological treatment in routine care. Also, our data on pharmacological drug use was extensively verified by review of the electronic patient files and by collection of drug delivery-data from local pharmacies. Furthermore, we extracted an ample amount of laboratory data on potassium and creatinine measurements prior to baseline to investigate the association between previous adverse events and current RAASi dosing.

This study also has some limitations. Because of the retrospective nature, the reasons not to choose maximum dose RAASi were not systematically registered in the patient files and neither was is information on side-effects. For the same reason, any relation between previous AKI or hyperkalemia and RAASi should be considered as hypothesis-generating. Additionaly, this was a single-center study performed in the Netherlands, therefore extrapolation to other populations should be done with caution. 
In conclusion, we found that in a real-life population of patients with type 2 diabetes in routine clinical care, only a third of patients used maximum dosage RAASi. Although the prevalence of contraindications for maximal RAASi, such a hyperkalemia and AKI, is considerable, they are usually relative in nature. These findings suggest that, to improve renoprotection and cardiovascular risk management in type 2 diabetes, it is worthwhile to explore strategies aimed at maximizing RAASi while circumventing the alleged contraindication.

\section{Declarations}

\section{Ethics approval and consent to participate}

The study has been approved by local institutional review boards (METC-Twente, NL57219.044.16; METCGroningen, 1009.68020), is registered in the Netherlands Trial Register (NTR trial code 5855) and is performed according to the guidelines of good clinical practice and the declaration of Helsinki as revised in 2008. All participants signed an informed consent form before participation.

\section{Consent for publication}

Not applicable

\section{Availability of data and materials}

The datasets generated and/or analysed during the current study are not publicly available due to ongoing analyses, but are available from the corresponding author on reasonable request.

\section{Competing interests}

The authors declare that they have no competing interests

\section{Funding}

We received an unrestricted clinical research grant from VIFOR for this work.

\section{Authors' contributions}

CG did the analyses wrote the main manuscript text en prepared the figures. CG, SHB and MO collected data. $\mathrm{SB}, \mathrm{GN}$ and GL provided materials. All authors reviewed the manuscript.

\section{Acknowledgements}

We would like to thank Roos Nijboer for her contribution in patients inclusion and data collection.

\section{References}

1. Cosentino F, Grant PJ, Aboyans V, et al. 2019 ESC Guidelines on diabetes, pre-diabetes, and cardiovascular diseases developed in collaboration with the EASD. Eur Heart J 2020; 41: 255-323.

2. Stevens PE, Levin A. Evaluation and management of chronic kidney disease: synopsis of the kidney disease: improving global outcomes 2012 clinical practice guideline. Ann Intern Med 2013; 158: 825-830. 
3. Knuuti J, Wijns W, Saraste A, et al. 2019 ESC Guidelines for the diagnosis and management of chronic coronary syndromes. Eur Heart J 2020; 41: 407-477.

4. Lewis EJ, Hunsicker LG, Clarke WR, et al. Renoprotective effect of the angiotensin-receptor antagonist irbesartan in patients with nephropathy due to type 2 diabetes. N Engl J Med 2001; 345: 851-860.

5. Brenner BM, Cooper ME, de Zeeuw D, et al. Effects of losartan on renal and cardiovascular outcomes in patients with type 2 diabetes and nephropathy. N Engl J Med 2001; 345: 861-869.

6. Parving $\mathrm{HH}$, Lehnert $\mathrm{H}$, Bröchner-Mortensen J, et al. The effect of irbesartan on the development of diabetic nephropathy in patients with type 2 diabetes. N Engl J Med 2001; 345: 870-878.

7. Vogt L, Laverman GD, de Zeeuw D, Navis G. The COOPERATE trial. Lancet 2003; 361: 1055-1056.

8. Ruggenenti P, Fassi A, llieva AP, et al. Preventing microalbuminuria in type 2 diabetes. N Engl J Med 2004; 351: 1941-1951.

9. Tran HA, Schwartzbard A, Weintraub HS. Role of RAAS Inhibition in the Prevention of Cardiovascular Disease. Curr Treat Options Cardiovasc Med 2011; 13: 279-288.

10. Taddei S. RAS inhibitors' dose-dependent efficacy: myth or reality?. Curr Med Res Opin 2015; 31: 12451256.

11. Blacklock CL, Hirst JA, Taylor KS, et al. Evidence for a dose effect of renin-angiotensin system inhibition on progression of microalbuminuria in Type 2 diabetes: a meta-analysis. Diabet Med 2011; 28: 11821187.

12. Taddei S, Bruno RM, Ghiadoni L. The correct administration of antihypertensive drugs according to the principles of clinical pharmacology. Am J Cardiovasc Drugs 2011; 11: 13-20.

13. Laverman GD, Henning $\mathrm{RH}$, de Jong $\mathrm{PE}$, et al. Optimal antiproteinuric dose of losartan in nondiabetic patients with nephrotic range proteinuria. Am J Kidney Dis 2001; 38: 1381-1384.

14. Laverman GD, Bakker SJL, Navis GJ. Optimal dosing of renin-angiotensin-aldosterone system blockers for renal protection: a solved issue?. Diabetologia 2009; 52: 1217-1218.

15. Epstein M, Reaven NL, Funk SE, et al. Evaluation of the treatment gap between clinical guidelines and the utilization of renin-angiotensin-aldosterone system inhibitors. Am J Manag Care 2015; 21: 212.

16. Gant CM, Binnenmars SH, Berg Evd, et al. Integrated Assessment of Pharmacological and Nutritional Cardiovascular Risk Management: Blood Pressure Control in the DIAbetes and LifEstyle Cohort Twente (DIALECT). Nutrients 2017; 9

17. Brar S, Ye F, James MT, et al. Association of Angiotensin-Converting Enzyme Inhibitor or Angiotensin Receptor Blocker Use With Outcomes After Acute Kidney Injury. JAMA Intern Med 2018; 178: 1681-1690.

18. Whelton PK, Carey RM, Aronow WS, et al. 2017 ACC/AHA/AAPA/ABC/ACPM/AGS/APhA/ASH/ASPC/NMA/PCNA Guideline for the Prevention, Detection, Evaluation, and Management of High Blood Pressure in Adults: A Report of the American College of Cardiology/American Heart Association Task Force on Clinical Practice Guidelines. Hypertension 2018; 71: e13-e115.

19. Williams B, Mancia G, Spiering W, et al. 2018 ESC/ESH Guidelines for the management of arterial hypertension. Eur Heart J 2018; 39: 3021-3104. 
20. Slagman MCJ, Waanders F, Hemmelder MH, et al. Moderate dietary sodium restriction added to angiotensin converting enzyme inhibition compared with dual blockade in lowering proteinuria and blood pressure: randomised controlled trial. BMJ 2011; 343: d4366.

\section{Figures}

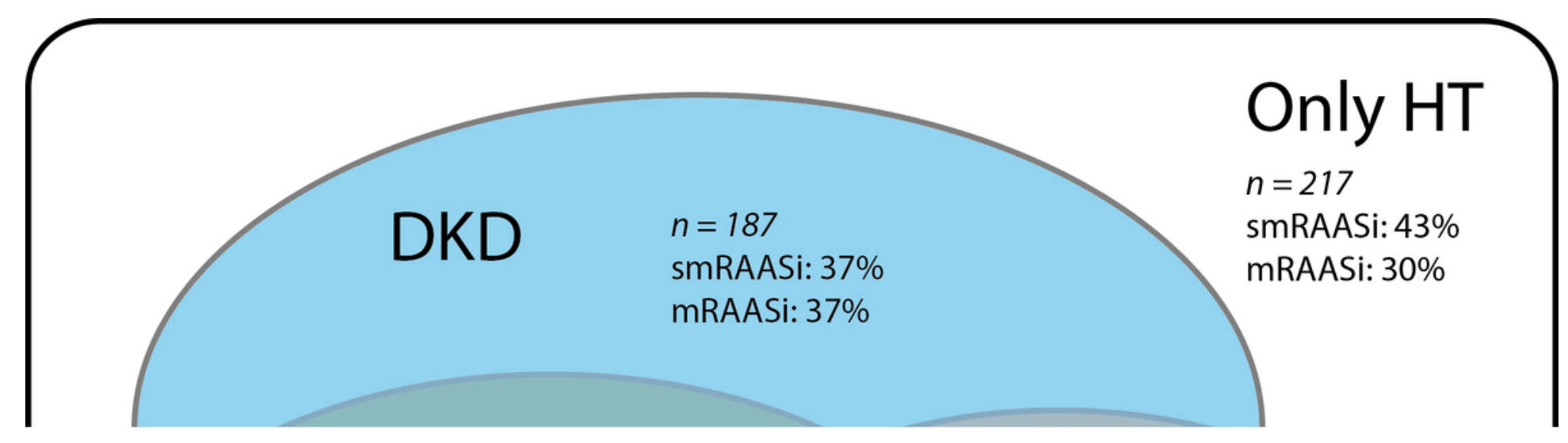

\section{Figure 1}

Proportion of patients using submaximal or maximal dosage of RAASi divided by indication. Patients with hypertension, diabetic kidney disease and coronary heart disease most often used maximal dosage RAASi.

DKD, diabetic kidney disease; CHD, coronary heart disease; TIA/CVA, cerebrovascular disease; HT, hypertension; smRAASi, use of submaximal dosage RAASi; mRAASi, use of maximal dosage RAASi.

\section{Figure 2}

Use of maximal dosage RAASi divided by number of times A) hyperkalemia and B) acute kidney injury in prebaseline measurements. 
noRAASi, no use of renin-angiotensin-aldosteron system inhibition; smRAASi, use of submaximal dosage RAASi; mRAASi, use of maximal dosage RAASi.

\section{Supplementary Files}

This is a list of supplementary files associated with this preprint. Click to download.

- OnlineOnlySupplementalMaterial.docx 